\title{
Critical fluctuations and breakdown of Stokes-Einstein relation in the Mode-Coupling Theory of glasses
}

\author{
Giulio Biroli ${ }^{1}$, Jean-Philippe Bouchaud ${ }^{2, \text {, }}$ \\ 1 Service de Physique Théorique Centre d'Études de Saclay \\ Orme des Merisiers, 91191 Gif-sur-Yvette Cedex, France \\ 2 Service de Physique de l'État Condensé, Orme des Merisiers, \\ CEA Saclay, 91191 Gif sur Yvette Cedex, France. \\ 3 Science \& Finance, Capital Fund Management, 6-8 Bd Haussmann, 75009 Paris, France.
}

(Dated: August 23, 2018)

\begin{abstract}
We argue that the critical dynamical fluctuations predicted by the mode-coupling theory (MCT) of glasses provide a natural mechanism to explain the breakdown of the Stokes-Einstein relation. This breakdown, observed numerically and experimentally in a region where MCT should hold, is one of the major difficulty of the theory, for which we propose a natural resolution based on the recent interpretation of the MCT transition as a bona fide critical point with a diverging length scale. We also show that the upper critical dimension of MCT is $d_{c}=8$.
\end{abstract}

Mode Coupling Theory ( $\mathrm{MCT}$ ) provides a useful theoretical framework to account for many of the empirical properties of liquids, at least in the weakly supercooled region 1, 2]. There are however a number of well known difficulties with the theory, the most prominent being the absence, in real systems, of the dynamical arrest singularity predicted by МСт. This very singularity underlies most of the quantitative predictions of MCT, such as the divergence of the relaxation time, the power-law shape of the correlator in the $\beta$ region, or the critical behaviour of the non-ergodic (Edwards-Anderson) parameter $q$ [1]. Additional activated relaxation processes, not described by MCT, have to be invoked to argue that the MCT transition temperature $T_{c}$ should be understood as a cross-over rather than a true singularity. In the region between $T_{c}$ and the so-called onset temperature, $T_{\text {onset }}$, where the slow dynamics regime sets in 31, MCT fares quite well to describe a host of experimental and numerical results with one noticeable exception: the breakdown of the Stokes-Einstein relation. This breakdown is one of the most important aspect of the phenomenology of supercooled liquid. The Stokes-Einstein relation states that the product of the viscosity $\eta$ times the self-diffusion constant $D$, divided by the temperature is a constant independent of temperature. This relation is well obeyed in liquids at high enough temperature. Instead, in supercooled liquids $D \eta / T$ increases quite shaply below $T_{\text {onset }}$ [4] and eventually reaches values of the order of 1000 or more at the glass temperature $T_{g}[5]$. In other words, the self-diffusion of particles becomes much faster than structural relaxation. The decoupling between viscosity and diffusion is interpreted by many to be a direct piece of evidence for dynamical heterogeneities in glassy liquids [5]. Intuitively, this decoupling comes about because diffusion is dominated by the fastest particles whereas structural relaxation is dominated by the slowest regions 5|. Detailed explanations based on different theoretical approaches have been put forward in the literature 6, 7, 8, 9]. In most models, the breakdown of the Stokes-Einstein relation is in fact a direct measure of the width of the distribution of local relaxation times (see below and $[\underline{5}, 6,6,6,8,9]$ ). Standard MCT does not account for the sharp increase of $D \eta / T$ between $T_{\text {onset }}$ and $T_{c}$ although this is precisely the region where MCT is supposed hold. The aim of this short contribution is to discuss an explicit mechanism that leads to such a breakdown within the mode-coupling theory of glasses, once critical fluctuations are taken into account.

In its usual interpretation, МCT describes homogeneous dynamics and neglects all fluctuations; it therefore seems that any effect associated to dynamical heterogeneities is outside of the scope of MCT. This pessimistic view was however recently argued to be erroneous [10, 11, 12, 13] (see [14, 15] for earlier insights). MCT should in fact be seen as a standard mean-field theory, except that it provides a self consistent equation for the two-body dynamical correlation function (describing density fluctuations) rather than for a one body order parameter, such as the magnetisation in the usual Curie-Weiss theory of magnets. In the latter case, we know that the appearance of a non trivial solution of the mean field equation below a certain critical temperature is in fact associated with the divergence of the magnetic susceptibility, itself related to magnetisation fluctuations. These fluctuations are harmless in high enough dimensions, but become dominant in dimension space less than four, where all critical properties of the phase transition are strongly affected by these fluctuations. The same scenario, although more involved, also holds for MCT 11]: above a certain dimension $d_{c}$, MCT is expected to be quantitatively accurate (at least in a regime where the above mentioned activated events can be neglected), whereas for $d<d_{c}$, dynamical fluctuations play a major role and must be properly accounted for. In this case, fluctuations of the (two body) dynamical correlation are measured through a four body correlation $G_{4}(\vec{r}, t)$; its integral over space define, in analogy with ferromagnets, a dynamical susceptibility called

*Electronic address: biroli@cea.fr,jean-philippe.bouchaud@cea.fr 
$\chi_{4}(t)$ in the recent literature [16, 17, 18]. The extension of MCT to compute $G_{4}(\vec{r}, t)$ reveals that $\chi_{4}$ indeed diverges as the mode-coupling temperature $T_{c}$ is approached 11, 12. This indicates that the dynamics becomes correlated over larger and larger length scales as the system freezes, which is in fact expected on general grounds: a diverging relaxation time should necessarily involve an infinite number of particles [19. The growth of $\chi_{4}$ has recently been detected in numerical simulations and experimentally in supercooled liquids [16, 17, 20] and in granular media [21]; it should also transpire in the divergence of the non-linear susceptibility of glassy systems 22].

As discussed in 11] the spatial correlations underlying the mode-coupling singularity necessarily lead to the existence of an upper critical dimension $d_{c}$ for this problem. In [11] it was shown that in the early $\beta$-relaxation regime, the four-point correlator reads, in Fourier space, $\hat{G}_{4}\left(\vec{k}, t \approx \tau_{\beta}\right) \sim\left(k^{2}+\sqrt{\epsilon}\right)^{-1}$, where $\epsilon=\left(T-T_{c}\right) / T_{c}$. From this, one can estimate, in the spirit of a Ginzburg argument [11], the fluctuations of the non-ergodic parameter $q$ in a region of size $\xi \sim \epsilon^{-1 / 4}$ to find $\xi^{d} \delta q \sim \xi^{d+2 / 2}$. Imposing that $\delta q$ must be much smaller than the critical behaviour predicted by MCT, i.e. $q_{c}-q \sim \sqrt{\epsilon}$, one finds that this assumption is only consistent when $d>d_{c}=6$. For $d<6$, the fluctuations become dominant for $\epsilon$ small enough and change all the critical exponents. But most interestingly, as we will argue below, these fluctuations are also responsible for the breakdown of the Stokes-Einstein relation. Before doing so, we should however point out that the analysis of [11] in fact overlooked a contribution due to the coupling between dynamic fluctuations and slow conserved degrees of freedom such as density and energy fluctuations [23]. These fluctuations, close enough to the transition, become the leading ones and change the above mean-field critical behaviour of $\hat{G}_{4}$ to $\hat{G}_{4}\left(\vec{k}, t \approx \tau_{\beta}\right) \sim\left(k^{2}+\sqrt{\epsilon}\right)^{-2}[23]$. In this case, the fluctuations of the non-ergodic parameter in a region of size $\xi$ grow as $\xi^{d+4 / 2}$, which becomes dominant below the critical dimension $d_{c}=8$ [38]. A diagrammatic derivation of this upper critical dimension is presented in the Appendix. Note that in reality the situation is more complicated: from numerical simulations of Lennard-Jones systems [23] one finds that fluctuations due to slow conserved degrees of freedom only become dominant very close to the transition, where MCT breaks down. For colloids, where MCT fares rather well, we do not have yet quantitative estimates of the relative contribution of these fluctuations. It might therefore well be that in cases of experimental or numerical interest, the original analysis of BB holds and the effective upper critical dimension is $d_{c}=6$. In any case, the aim of this paper is not to obtain quantitative predictions but only to showing that critical dynamical MCT fluctuations provide a natural scenario for the decoupling of self-diffusion and viscosity.

In principle, the contribution of critical fluctuations to the viscosity and diffusion constant should be calculated using a renormalisation group around $d_{c}$. Here, we do not attempt to do this but argue physically that such a program should lead to a decoupling between these two quantities. We assume that non trivial critical exponents describe, in $d<d_{c}$, the different physical quantities; for example $\xi \sim \epsilon^{-\nu}, q_{c}-q \sim \epsilon^{\beta}, \tau \sim \epsilon^{-\gamma}$ and $G_{4}(\vec{r}) \sim g(r / \xi) / r^{d-4+\eta}$. Modecoupling theory describes how the random potential created by density fluctuations slows down the particles. Close to the MCT transition, this self-generated random potential persists on a timescale comparable to the one responsible for structural relaxation. Hence, it acquires a static component that self-consistently traps the particles at the MCT transition, where the relaxation timescale diverges. The strength of this self-generated component, and hence of the trapping potential, is measured by the plateau value $q$ of the correlation function, which therefore acts as an effective coupling constant. When $q$ reaches $q_{c}$, the random potential is sufficiently strong to prevent the particles from moving. Clearly, activated effects are expected to destroy the transition (any finite potential barrier can be overcome in finite time), but within MCT, these processes are neglected.

The local difference between $q$ and $q_{c}$ therefore plays a major role: if the local value of $q$ is slightly smaller, particles are less trapped and diffusion is enhanced. If, on the other hand, the local value of $q$ is slightly larger, particles are frozen [39]. In other words, the self-generated disorder fluctuates in space and leads to fluctuations of the critical temperature on correlated regions of linear size $\xi[40$. Since the (local) relaxation time is set by the distance from the (local) critical temperature, the dynamics strongly fluctuates from one correlated region to another. This is precisely why the fluctuations of the local correlation function, measured by $G_{4}(\vec{r}, t)$, play a crucial role to generate strong dynamical heterogeneities. Parallel to the Ginzburg argument, the fluctuations (per particle) of the non-ergodic parameter in a region of size $\xi \sim \epsilon^{-\nu}$ are given by $\delta q \sim \xi^{(4-d-\eta) / 2} \sim \epsilon^{-\nu(4-d-\eta) / 2}$, to be compared with $q_{c}-q \sim \epsilon^{\beta}$. These fluctuations of the effective coupling constant in turn induce local fluctuations of the relaxation time as:

$$
\frac{\delta \tau}{\tau}=\frac{\gamma}{\beta} \frac{\delta q}{q_{c}-q} \sim \epsilon^{\frac{\nu(d+\eta-4)}{2}-\beta} .
$$

For $d>d_{c}$, the mean field value of the exponents $\nu=1 / 4, \beta=1 / 2$ can be used, and one finds that $\delta \tau / \tau \rightarrow 0$ close to the MCT transition: dynamical heterogeneities are mild and do not jeopardize the Stokes-Einstein relation, as predicted by МCT calculations that neglect spatial fluctuations altogether. Interestingly, this conclusion does not hold for $d<d_{c}$, where the relative width of the local relaxation time distribution must increase as $\epsilon \rightarrow 0$. For $\epsilon$ not too small, one can still use the mean-field value of the exponents, but now the fluctuations of local relaxation times are found to increase as $\delta \tau / \tau \sim \epsilon^{-\left(d_{c}-d\right) / 4}$, before one enters the Ginzburg region, where fluctuations eventually change 
the values of the exponents. For standard phase transitions, the hyperscaling relation $\nu(d+\eta-2)-2 \beta=0$ is such that fluctuations remain of order one when $\epsilon \rightarrow 0$. Its generalization to the MCT transition would be $\nu(d+\eta-4)-2 \beta=0$. However, it is not clear whether within MCT hyperscaling holds since the MCT transition is purely dynamical, and has a mixed first order/second order nature $\left(q_{c}\right.$ is non zero at $T=T_{c}$ while the relaxation time is diverging as a power law). A detailed renormalisation group study of the replica field theory with a cubic term could help settling this interesting technical point. In any case, we expect that in three dimensions, neglecting the role of the activated processes (that cut off the transition) and entering in the regime where the dynamics is supposed to slow down because of the MCT critical point (i.e. between $T_{\text {onset }}$ and $T_{c}$ ) $\delta \tau / \tau$ should first grow as $\epsilon$ decreases, and either saturate or diverge as $\epsilon \rightarrow 0$, depending on the validity of hyperscaling.

As already discussed, a key ingredient that generates violation of the Stokes-Einstein relation is the fluctuation of the local relation timescale $\tau$. Different models capture this effect. For instance, assuming as in 7] local fluctuations of the diffusion coefficient one finds that the Stokes-Einstein violation is related to the ratio between $\langle 1 / \tau\rangle$ and $1 /\langle\tau\rangle$. Another model consists in assuming that particles hop with random relaxation times. In this case the self diffusion coefficient is given by $D=\ell^{2} /\langle\tau\rangle$, where $\ell$ is the typical hopping distance, expected to be of the order of the particle size and roughly temperature independent. The viscosity, or the terminal relaxation time, on the other hand, are given by the integral of the average correlation function, which weighs slow regions proportionally to the local relaxation time; therefore $\eta \sim\left\langle\tau^{2}\right\rangle /\langle\tau\rangle$. In both models the crucial ingredient to evaluate violation of the Stokes-Einstein relation are indeed local fluctuations of the relaxation times. For example for the latter model, one gets:

$$
\frac{D \eta}{K_{S E} T} \propto 1+\left\langle(\delta \tau)^{2}\right\rangle /\langle\tau\rangle^{2} \sim 1+C \epsilon^{\nu(d+\eta-4)-2 \beta},
$$

where $C$ is a numerical constant of order unity and $K_{S E}$ is the high temperature value of $D \eta / T$. For $d<d_{c}$, this formula predicts an increasing decoupling between viscosity and diffusion between $T_{\text {onset }}$ and $T_{c}$. If $C$ is not too small, we predict a power-law viscosity-diffusion decoupling as $T_{c}$ is approached, with exponents that change as one enters the Ginzburg region. Such a behaviour may have been observed in numerical simulations of Lennard-Jones particles [2], where $D \eta / T$ is found to increase by a factor $\sim 5$ in the MCT region [4], while MCT predicts an increase of a mere $1.2[2,29]$. These results are interesting because they show that both the viscosity and the inverse diffusion constant can be made to scale relatively well with $\epsilon=\left(T-T_{c}\right) / T_{c}$ with the same $T_{c}$ for both quantities, but with different exponents. In other words, this scenario is compatible with a 'fractional' Stokes-Einstein relation, $D \sim \eta^{-k}$ with $k<1$. More precisely, the numerical results are $\eta \sim \epsilon^{-2.4}$, whereas $D \sim \epsilon^{1.8}$, so that $D \eta \sim \epsilon^{-0.6}$ (see [4] and refs therein). These results suggest that the observed breakdown of the Stokes-Einstein relation might indeed be related to critical fluctuations. A similar breakdown of the Stokes-Einstein relation in the mode coupling region has also been observed in hard sphere systems, both experimentally [26] and numerically [29, 30]. Of course, much stronger violations are observed closer to $T_{g}$, but outside the region where MCT can be valid. These should be related to the broadness of activated relaxation time distributions and are outside the scope of the present discussion [41].

Summarizing this short contribution, we have argued that a dramatic consequence, in low space dimensions, of the critical dynamical fluctuations predicted by the mode-coupling theory of glasses is the breakdown of the StokesEinstein relation. This breakdown, observed numerically and experimentally in a region where MCT should hold, i.e. above $T_{c}$, is one of the major difficulty of MCT (setting aside other well known difficulties related to activated processes), and for which we provide a natural interpretation. We believe that this matter deserves more investigation, both

- theoretically, to estimate the value of the exponents describing this breakdown in $d<d_{c}$ and analyze the interplay between activated processes and critical dynamical fluctuations;

- and numerically and/or experimentally to ascertain the connection between dynamical fluctuations and viscositydiffusion decoupling in the $T>T_{c}$ region, for example by comparing the location $\tau$ and the width $\delta \tau$ of the peak of the four-point susceptibility $\chi_{4}(t)$.

Numerically one could also test the dimension dependence of Stokes-Einstein violation, and compare with other scenarii, such as suggested by Kinetically Constrained Models which also predict such violations [31]. If confirmed, those effects would be a direct proof of the importance of critical fluctuations in glasses. This would put the program of extending MCT using renormalisation group methods on top of the agenda.

We thank L. Berthier, D. R. Reichman, R. Richert and M. Wyart for many useful discussions on these topics. GB is partially supported by EU contract HPRN-CT-2202-00307 (DYGLAGEMEM). 


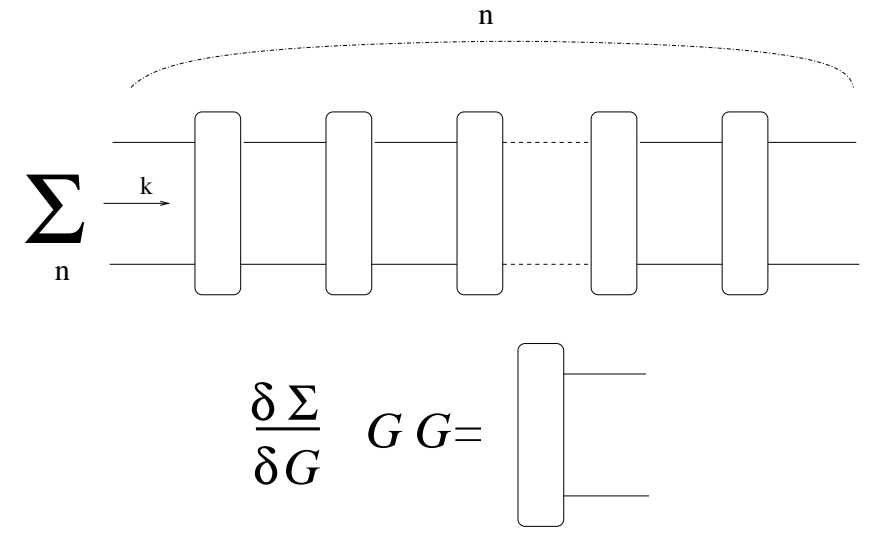

FIG. 1: Diagrammatic representation of the parquet diagrams. $\vec{k}$ denotes the wave-vector entering into the ladders.

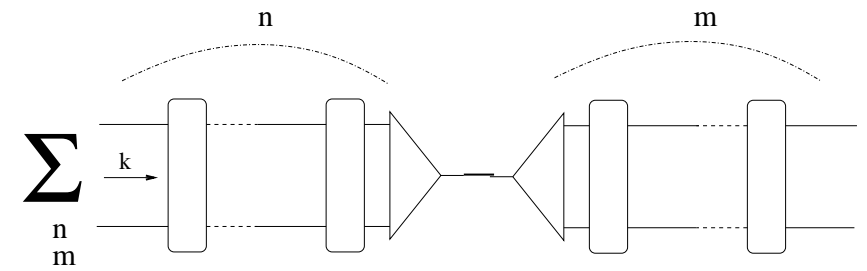

FIG. 2: Diagrammatic representation of the squared-parquet diagrams. $\vec{k}$ denotes the wave-vector entering into the ladders.

\section{Appendix}

In the following we shall show that below eight dimensions there are infrared divergences that change the mean-field scaling derived in $\mathrm{BB}$ and $[23]$.

The natural framework to analyze critical dynamical fluctuations and their role in determining the upper critical dimension is dynamical field theory. The starting point to analyze the dynamics of super-cooled liquids consists in writing down some exact or phenomenological stochastic equations for the evolution of the slow conserved degrees of freedom. Field-theories are obtained through the Martin-Siggia-Rose-deDominicis-Janssen method, where one first introduces response fields enforcing the correct time evolution and then averages over the stochastic noise [33]. Standard MCT corresponds to a self-consistent one-loop approximation (see Refs. [34, 35, 36] for a discussion of the different field-theories and subtleties appearing in the derivation of MCT).

Here we do not provide any detail and we refer to [23, 34] for a thorough presentation. We only recall that generically the four-point function can be written in terms of the so called parquet diagrams, see [37] for a general introduction and 23] for an application to supercooled liquids. As shown in 23] the four-point function can be exactly expressed as a sum of parquet and "squared-parquet" diagrams (we will use the same notation of [23]), see Fig. 1] and Fig. 2]

The elementary block used to construct the parquet diagrams corresponds to $[\delta \Sigma / \delta G] G G$ where $\Sigma$ is the self-energy considered as a function of the dressed propagator $G$, see 23]. MCT consists in retaining only the bubble diagram for the self-energy. In this case the elementary block is very simple and leads to the ladder diagrams studied in BB and [23], which lead to the mean-field scaling discussed in the main text. The diagram in Fig. 1] gives a contribution to $G_{4}(k, t)$ that, for times $t$ in the $\beta$ regime and for small $k$, has a critical behavior like $1 /\left(k^{2}+\sqrt{\epsilon}\right)$. The diagram in Fig. 2] on the other hand, gives a contribution that has a critical behavior like $1 /\left(k^{2}+\sqrt{\epsilon}\right)^{2}$.

From the point of view of critical phenomena, one expects that adding diagrams other than the bubbles to the self-energy should be harmless and should not change the scaling for all dimensions larger than the upper critical dimension $d_{c}$. In order to show that this is the case and determine $d_{c}$ one has to prove that (1) adding any type of diagram to the bubbles does not change the MCT (mean-field) critical properties in presence of an infrared cut-off on momenta integration and (2) equating the infrared cut-off to zero leads to a change of the mean-field scaling only below the upper critical dimension $d_{c}$. The first part of this procedure is lengthly and difficult and it will be shown elsewhere [32]. Concerning the second part, we shall only show an example of diagrams that are responsible for changing the mean-field scaling below eight dimension. (A full analysis showing that above eight dimensions all diagrams are harmless is outside the scope of this article and is left for future work).

In order to show an example of divergent diagram below eight dimension one has to focus on the elementary block 


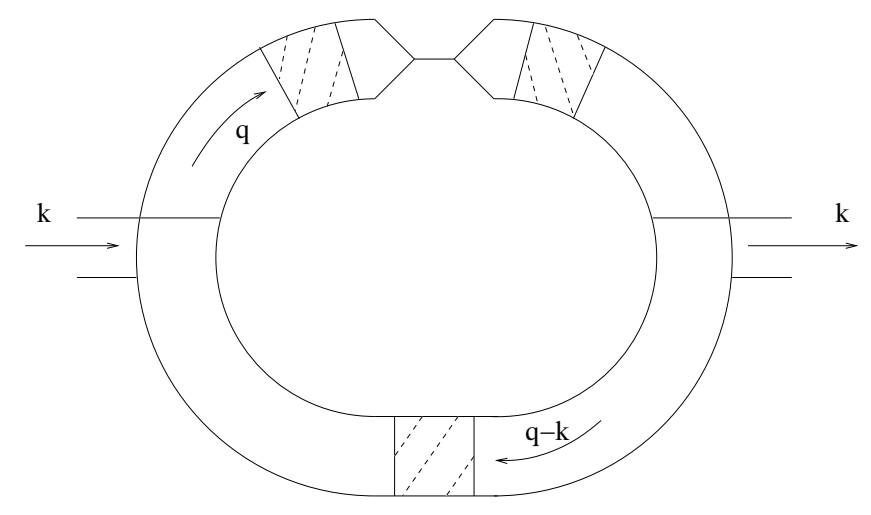

FIG. 3: Divergent diagram below eight dimension assuming mean-field scaling. One box represents the parquet diagram shown in Fig. 1 Note that the lines on the left have to be considered without propagators.

$[\delta \Sigma / \delta G] G G$ used to construct the parquets. The small $k$ behavior of this block determines the MCT mean-field scaling discussed above ( $k$ is the momentum entering into the block as shown in Fig. 1 and 2). Within MCT, and more generally, if one puts an infrared cutoff, one finds a scaling $k^{2}+\sqrt{\epsilon}$ at small $k$. One can show that this assumption is inconsistent in low enough dimension, focusing on an explicit diagram, see Fig. 3 [42].

In order to do that, we shall assume that the mean-field scaling is correct and compute the small $k$ behavior of this diagram. In dimensions less than $d_{c}$, the corresponding contribution to $[\delta \Sigma / \delta G] G G$ at small $k$ will be found to differ from mean-field. For the diagram in Fig. 3 the small $k$ dependence is given by (we omit the dependence on the non-critical wave-vectors):

$$
I(k, \epsilon) \sim \int \frac{1}{\left(q^{2}+\sqrt{\epsilon}\right)^{2}} \frac{1}{(q-k)^{2}+\sqrt{\epsilon}} d^{d} q
$$

For $\epsilon=0$ the small $k$ behavior is then given by $I(0,0)+c k^{2}+\ldots$ where $I(0,0)$ and $c$ are two well-defined constants above eight dimensions (that can be explicitely computed from $I(k, \epsilon)$ ). $I(0,0)$ and $c$ lead, respectively, to a renormalization of the critical temperature and of the "rigidity" constant in front of the $k^{2}$ term in $G_{4}$. However, below eight dimensions, $c$ diverges (if the infrared cutoff vanishes) and the behavior of $I(k, 0)$ is singular at small $k: I(0,0)+c^{\prime} k^{2-(8-d)}+\ldots$ This clearly shows that the assumption of mean field scaling is not correct. In order to go further and compute the non mean field exponents one should perform a renormalization group treatment, or some self-consistent (mode-coupling!) resummation of these dangerous diagrams. We leave this for further investigations.

As a conclusion we find, in agreement with the simple Ginzburg argument developed in the main text, that below eight dimension critical fluctuations are not governed by mean-field theory.

Finally, we remark that for a system with no conserved degrees of freedom, as it is the case for the Langevin dynamics of disordered p-spin systems, the squared-parquet diagrams are absent. Therefore the divergent diagram corresponding to the one in Fig. 3 is now formed by single parquets both on the top and bottom parts of the diagram. Repeating the same analysis performed above, one finds $d_{c}=6$ in this case, as found in BB.

[1] W. Götze, L. Sjögren, Rep. Prog. Phys. 55241 (1992); W. Götze, Condensed Matter Physics, 1, 873 (1998); W. Götze, J. Phys.: Condens. Matter 11 A1-A45 (1999).

[2] W. Kob, H. C. Andersen, Phys. Rev. E 514626 (1995); 524134 (1995); see also: W. Kob, in Slow relaxations and non-equilibrium dynamics in condensed matter, Les Houches, Session LXXVII, J. L. Barrat, M. Feigelman, J. Kurchan, J. Dalibard Edts, Springer-EDP Sciences (2003).

[3] S. Sastry, P. G. Debenedetti and F. H. Stillinger, Nature 393, 554 (1998).

[4] P. Bordat, F. Affouard, M. Descamps and F. Muller-Plathe, J. Phys. Cond. Matt. 155397 (2003).

[5] see e.g. M. D. Ediger, Ann. Rev. Phys. Chem. 51, 99 (2000).

[6] F. H. Stillinger, J. Chem. Physics 89, 6461 (1988); F. H. Stillinger and J. A. Hodgdon Phys. Rev. E 502064 (1994).

[7] G. Tarjus, D. Kivelson, J. Chem. Phys. 1033071 (1995).

[8] X. Xia and P. G. Wolynes, J. Phys. Chem. B, 105, 6570 (2001).

[9] Y. Jung, J.P. Garrahan and D. Chandler, Phys. Rev. E 69, 061205 (2004).

[10] S. Franz, G. Parisi, J. Phys.: Condens. Matter 12, 6335 (2000); C. Donati, S. Franz, G. Parisi, S. C. Glotzer, J. Non-Cryst. Sol., 307, 215-224 (2002). 
[11] G. Biroli, J. P. Bouchaud, Europhys. Lett. 67, 21-27 (2004).

[12] G. Biroli, J.P. Bouchaud, K. Miyazaki, D. R. Reichman, Inhomogeneous Mode-Coupling Theory and growing dynamic length in supercooled liquids, cond-mat/0605733

[13] S. Franz, A. Montanari, Dynamical and mosaic length scales in a Kac glass model, cond-mat/0606113

[14] T.R. Kirkpatrick, P.G. Wolynes, Phys. Rev. B 36, 8552 (1987).

[15] T.R. Kirkpatrick and D. Thirumalai, Phys. Rev. A 37, 4439 (1988).

[16] C. Bennemann, C. Donati, J. Bashnagel, S. C. Glotzer, Nature, 399, 246 (1999), S. C. Glotzer, J. Non-Cryst. Solids 274, $342(2000)$.

[17] L. Berthier, Phys. Rev. E 69, 020201/1-4 (2004); S. Whitelam, L. Berthier, J.P. Garrahan, Phys. Rev. Lett. 92, 185705/1-4 (2004).

[18] C. Toninelli, M. Wyart, G. Biroli, L. Berthier, J.P. Bouchaud, Phys. Rev. E 71, 041505/1-20 (2005).

[19] A rigorous proof for a large class of glassy systems has been presented in A. Montanari, G. Semerjian, Rigorous Inequalities between Length and Time Scales in Glassy Systems, cond-mat/0603018

[20] L. Berthier, G. Biroli, J.-P. Bouchaud, L. Cipelletti, D. El Masri, D. L'Hote, F. Ladieu, M. Pierno, Science 310, 1797 (2005).

[21] O. Dauchot, G. Marty and G. Biroli, Phys. Rev. Lett. 95, 265701 (2005).

[22] J. P. Bouchaud, G. Biroli, Phys. Rev. B 72064204 (2005).

[23] L. Berthier, G. Biroli, J.P. Bouchaud, W. Kob, K. Miyazaki, D. R. Reichman, cond-mat/0609656 and 0609658.

[24] A. Widmer-Cooper and P. Harrowell, Predicting the long time dynamic heterogeneity in a supercooled liquid on the basis of short time heterogeneities, e-print cond-mat/0512035

[25] A. Aharony and A.B. Harris, Phys. Rev. Lett. 773700 (1996); A. Aharony, A.B. Harris and S. Wiseman Phys. Rev. Lett. 81252 (1998); S. Wiseman and E. Domany, Phys. Rev. Lett. 8122 (1998).

[26] D. Bonn, W. Kegel, J. Chem. Phys. 1182005 (2003)

[27] R. A. Denny, D. R. Reichman, and J. P. Bouchaud, Phys. Rev. Lett. 90025503 (2003).

[28] B. Doliwa, A. Heuer, Phys. Rev. E 67 030501(R) (2003)

[29] Th. Voigtmann, A. M. Puertas, M. Fuchs, Phys. Rev. E 70, 061506 (2004).

[30] S. Kumar, G. Szamel, J. Douglas, Nature of the Breakdown in the Stokes-Einstein Relationship in a Hard Sphere Fluid, cond-mat/0508172

[31] see e.g. Y. Jung, J.P. Garrahan and D. Chandler, Phys. Rev. 69061205 (2004); preprint cond-mat/0504535 L. Berthier, D. Chandler, and J.P. Garrahan, Europhys. Lett. 69230 (2005).

[32] A. Andreanov, G. Biroli, J.-P. Bouchaud, in preparation.

[33] J. Zinn Justin, Quantum field theory and critical phenomena (Oxford University Press, Oxford, 2002).

[34] A. Andreanov, G. Biroli, and A. Lefèvre, J. Stat. Mech. P07008 (2006).

[35] S. P. Das, Rev. Mod. Phys. 76, 785 (2004).

[36] K. Miyazaki and D. R. Reichman, J. Phys. A: Math. Gen. 38 (2005) L343.

[37] J.-P. Blaizot, G. Ripka, Quantum Theory of Finite Systems, Editions Phenix, Kiev, 1998.

[38] The analysis of [11] is strictly speaking correct for models that are characterized by a dynamics without conserved quantities. An example is a finite dimensional p-spins model for which the upper critical dimension is therefore $d_{c}=6$.

[39] This means that the local Debye-Waller factor is expected to be strongly correlated by the particles' propensity to diffuse, as indeed recently found numerically in [24], and L. Berthier, unpublished.

[40] see 25] for a discussion of the role of critical temperature fluctuations in disordered systems.

[41] Note however that activated events play a role at long times even above $T_{c}$, and might thus be at least partly responsible for the decoupling [27, 28].

[42] Note that the diagram shown in Fig. 3] is composed of a double parquet on the top and a single parquet on the bottom. It is not possible to have double parquet both on the top and bottom parts because joining the two lines on the right and on the left one must construct a two particle irreducible diagram, and this diagram could be cut in two pieces just cutting the two middle lines. 